\title{
Hybrid Approach for Automatic Drug Dispensing and Control
}

\author{
NAVEEN KUMAR ${ }^{1}$, S.N.PANDA ${ }^{2}$, PREETHI PRADHAN ${ }^{3}$ \\ ${ }^{1}$ Chitkara University, Punjab, India \\ ${ }^{2}$ Chitkara University, Punjab, India \\ ${ }^{3}$ Chitkara University, Punjab, India
}

\section{Emial: preethi@chitkara.edu.in}

Received: December 18, 2016 I Revised: February 08, 2017| Accepted: March 02, 2017

Published online: April 10, 2017

The Author(s) 2017. This article is published with open access at www.chitkara.edu.in/ Publications

\begin{abstract}
Clinical scenario for critically ill patients involves large number of medical devices, such as, bed side monitors that provide vital information about the admitted patient. Critically ill patients need prompt and perfect decision, so that lifesaving drugs can be delivered at a proper time. Moreover suggestions/ recommendations of a super specialist doctor may also be required in hospitals. The main problem in hospitals is lack of specialist doctors, so, patients are not able to approach to them on time. Automatic drug delivery using microprocessor/ microcontroller has improved tremendously in recent years due to advancement in Information and Communication Technology (ICT). This paper is focused on various Drug Delivery System Interfaces used in emergency cases to reduce manual intervention. We are proposing a hybrid approach for drug dispensing, which can work as open or closed loop system. Another feature of this system is that it can be controlled from a remote location, so is to provide virtual presence of a doctor. The system will help the doctor monitor the patient as per the immediate requirement and deliver drug remotely to patient using smart phone.
\end{abstract}

Keywords: Automatic Drug delivery, Infusion pump, Open Loop, Closed Loop, Depth of Anaesthesia, BIS.

\section{INTRODUCTION}

The primary goal of Health Professionals should be the provision of the best possible quality care of the patient. This becomes possible due to automated medical devices which are equipped with microprocessors/ controller/ sensors. Automatic Drug Delivery System using infusion pumps/ drug dispenser which

\footnotetext{
Journal of

Multidisciplinary

Research in Healthcare

Vol-3, No-2,

April 2017

pp. 69-77
} 
Kumar, $\mathrm{N}$

Panda, SN

Pradhan, $\mathrm{P}$

come with controllers to control hypnosis has improved tremendously in recent years. Automated drug delivery system is controlled by pharmacodynamics feedback known as closed loop system performs better in comparison with model base (open loop system). Thus, drug delivery is a technique which is used to control the pharmaceutical compounds in human beings to administer the therapeutic effects. The prime role of health science is to provide best care to the patients [2]. An extensive literature review was conducted using the terms: open loop, closed loop, automatic drug delivery system, distant monitoring and vital sign monitoring. In this research paper a survey was conducted to collect data from doctors. This survey was aimed to design the architectural model of the drug delivery system capable of delivering accurate amount of drug. The subsequent part of the paper is divided in sections: in the next section we discuss about Manual Drug Delivery System. In section III Automatic Drug Delivery System is introduced. In section IV Open Loop Drug Delivery is discussed. Section V is about closed loop drug delivery system. Section VI is about outcome of the survey and final section presents conclusion and future work

\section{MANUAL DRUG DELIVERY SYSTEM}

It is a type of traditional delivery method still used by doctors. Routes for manual drug deliveries may be different like mouth, skin, nasal and inhalation etc. The major drawback of this type of drug delivery is that the probability of miscalculation and mishandling is more. So far, this method of drug delivery is used only by doctors who don't want to use a fully automatic system that can take the decision on its own. Therefore, this model of drug delivery is used in majority of the primary hospitals. On the other hand, this method has its own drawbacks like miscalculation, over-dosing and/or under-dosing. For example, in anaesthesia, under-dosing can cause severe pain and over-dosing may cause late recovery of the patient after the surgery [7]. Manual drug delivery is not suitable in situation where dose of a drug is to be given at a regular dosing interval. In many situations, there is a need to deliver drug at a very low rate e.g. $5 \mathrm{ml} /$ hour. Hence through manual drug delivery approach, it is not possible to deliver a fixed amount of drug in a given time interval. In such kind of situations, Automated Drug Delivery Systems consisting of microprocessor/ controllers are preferred.

\section{AUTOMATIC DRUG DELIVERY SYSTEM}

Automated Drug Delivery System has proven more advantageous as compare to Manual Drug Delivery Systems [9]. Automatic Drug Delivery due to 
advance microprocessors/ controllers has enhanced tremendously in recent years. Automatic Drug Delivery System (ADDS) comprises an infusion pump with a controller, actuator, and stepper motor. The imperative functionality of the infusion pump is to release precise amount of a drug in prearranged time. Major components of an infusion pump are controller, stepper motor and actuator.

Controller: A Controller inside an infusion pump is an integrated chip with memory; hence it can be programmed to rotate a stepper motor by giving appropriate instructions either in forward or reverse direction. It can be programmed with drug delivery algorithm that performs an action which enables it to steer the control variable [10].

Stepper Motor: A stepper motor is one of the major components of an infusion pump that gets instruction from microcontroller and moves accordingly. The accuracy of a stepper motor depends upon the number of steps it has. The higher is the number of steps, higher is the accuracy. Usually, bipolar stepper motor is used inside the infusion pump.

Actuator: Actuator is the most import mechanical part inside the infusion pump that converts rotary motion of the motor into linear motion Automatic Infusion pump is a pre-programmed electromechanical device that can be operated either in open loop or closed loop as per the requirement. Therefore, we are proposing a hybrid kind of drug delivery system that can be operated both in open or closed loop as per the requirement with GUI (Graphical User Interface) and minute to minute database storage. This database will be helpful to the researchers to analyze the detail case study in future. Remote access is one of the key features of this Hybrid Drug Delivery System. In simpler terms remote access is one of the key feature of this hybrid drug delivery system that may enable a specialist doctor to view patient data on his/her smart phone.

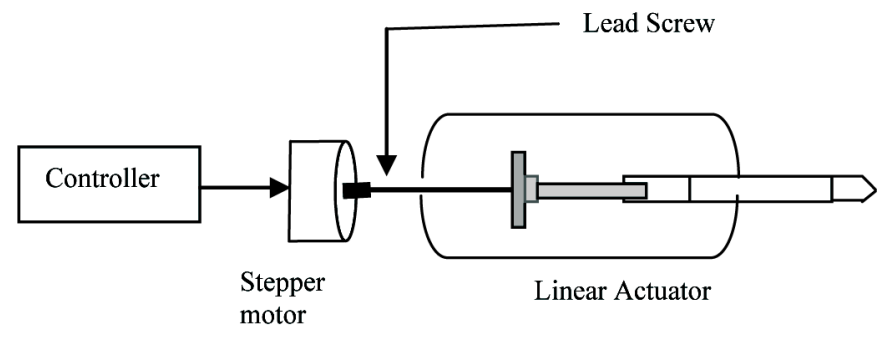

Hybrid Approach for Automatic Drug

Dispensing and Control

.

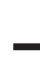

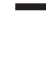

(n)


Kumar, $\mathrm{N}$

Panda, SN

Pradhan, $\mathrm{P}$

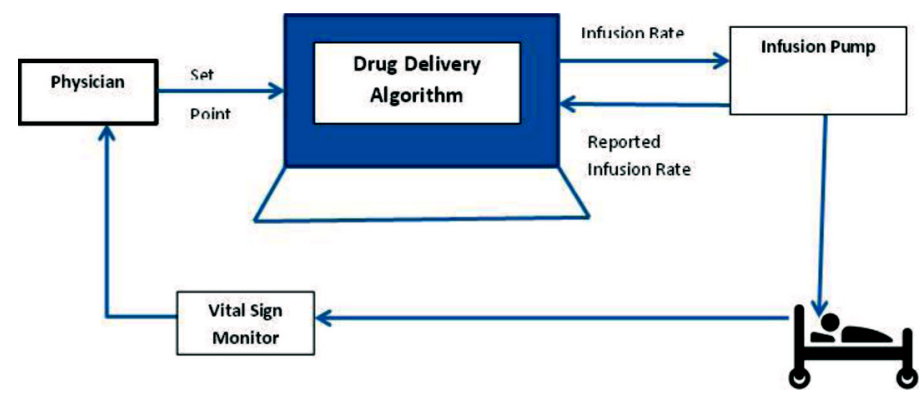

Figure 2: Open Loop Drug Delivery System.

\section{OPEN LOOP DRUG DELIVERY SYSTEM}

Open loop or model base drug delivery system used by clinical personnel, can be tedious, imprecise, time-consuming and sometimes of poor quality depending upon the skills and judgment of clinician [1]. Although underdosing and over-dosing can lead to detrimental health consequences for the patient. The performance of open-loop system is highly dependent on the accuracy of the model it is based upon [6]. Van Halteren et al. developed MobiHealth with the help of Body Area Network (BAN) to transmit patient data remotely MobiHealth does not support automated monitoring and patient feedback [14].

Working of Open Loop: Both open and closed loop drug delivery system require a set point initially as an input to the drug delivery algorithm. Algorithm calculates the amount of drug and delivered it to patient through infusion pump. In case there are changes in the vital signs of the patient, clinician may change the rate of the drug as per his/her expertise.

\section{CLOSE LOOP DRUG DELIVERY SYSTEM}

From the beginning of the Eighties Physicians and Engineers have been trying to develop a closed-loop controller for drug delivery. One of this forms of drug delivery was developed by Koivo for blood-pressure control system that was tested on dogs [3]. Closed loop drug delivery system is a form of Automatic Drug Delivery System which works on feedback mechanism as shown in figure-3. The feedback signal is the value that has been resulted from the automated delivery system. A Closed Loop System senses the level of output compares and sends this information as feedback to a set point. This feedback is known as control variable. To measure depth of anesthesia EEG is often used by anesthetist [8] and [11]. Therefore, a vital sign monitor is used to view the control variable is always required to assist the clinician [4]. 


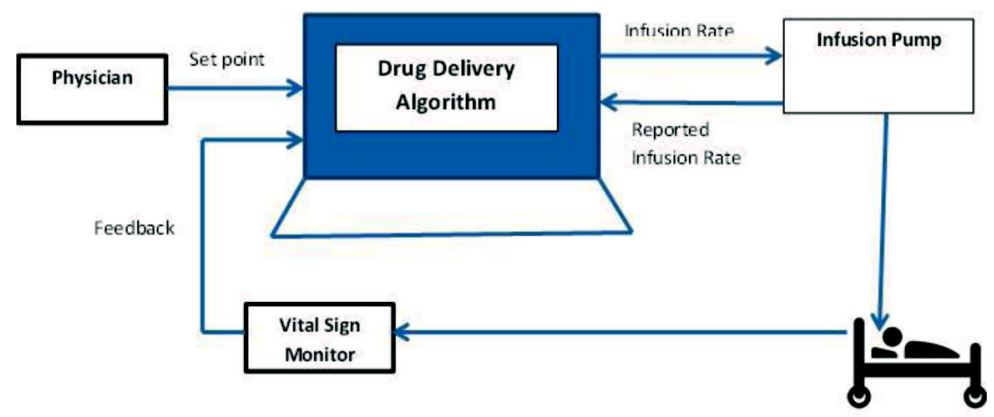

Hybrid Approach for Automatic Drug

Dispensing and Control

Figure 3: Hybrid Drug Delivery System.

A drug delivery system with feedback controller cans perform much better as compared to a Open Loop System. A controller is far more powerful as compared to the same for human because a feedback mechanism can decrease the chances of uncertainty [6]. Burno et al proposed MOHLL (Mobile Health Living Lab) that transmits ECG (Electrocardiography), SPO2 (Oxygen Saturation) and body temperature using microcontroller (ZigBee) to a data server [13]. Bingchuan et al. proposed Web based Real Time monitoring System, CARA (Context-Aware Real-Time Assistant) that may be used to analyse real time vital parameters of a patient and home monitoring system for self-governance of elderly persons [15]. Feed-back guided CLADS provided clinically adequate amount of drug and satisfactory operating conditions in all studies [9]. Closed loop systems have an advantage over manual drug delivery system which can be a precious support to control hemodynamic.

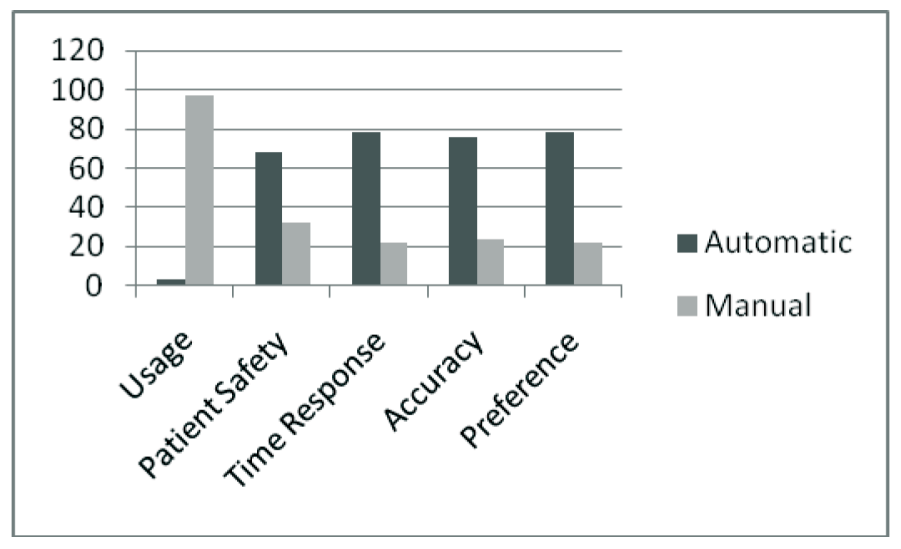

Table 1: Comparison Between Automatic And Manual. 


\begin{tabular}{lll}
\cline { 2 - 3 } $\begin{array}{l}\text { Kumar, } \mathrm{N} \\
\text { Panda, SN } \\
\text { Pradhan, } \mathrm{P}\end{array}$ & \multicolumn{1}{c}{ Close Loop } & \multicolumn{1}{c}{ Open Loop } \\
\cline { 2 - 3 } & During Surgery & $\begin{array}{l}\text { Post Operatively and critically ill } \\
\text { patients }\end{array}$ \\
& Hemodynamic Unstable & ICU \\
& Metabolic Disorder & Shock \\
& Under Supervision & Critically ill Patients in ICU/ \\
& CCU \\
& Post Operative Patients & Renal Failure \\
\hline
\end{tabular}

Table 2: Usages of Close and Open Loop.

\section{OUTCOME OF THE SURVEY}

A survey was conducted in tertiary and multispecialty hospitals to get the current Drug Delivery Type (DDT) used by doctors and their willingness to adopt the drug delivery system, which gets them better and quick results. Sample size of this survey was 40 . The average experience of doctors from whom the data had been collected was 12 years. The survey was aimed at objectifying the need of Drug Delivery Systems which is capable to deliver accurate amount of drug and to design the architectural model. From the survey it was found that $97 \%$ of doctors are using manual drug delivery system, but when they were asked about patient safety, and time response, they believes that automatic drug delivery system is better than manual drug delivery system.

When they were asked about patient safety $32 \%$ of respondents felt that there was accuracy in the the Manual Drug Delivery System, whereas 68\% trusted in automatic drug delivery system. Doctors also believed that there was more accuracy in the automatic drug delivery system.

Doctors were also asked about their willingness to control the system from remote location. As per the outcome of the survey, $84 \%$ of doctors wished to use a system that can be controlled from a remote location, which may provide virtual presence of a doctor. The outcome depicts that although presently $97 \%$ of doctors are using manual drug delivery system but they wished to use an Automatic Drug Delivery System. So, a hybrid system can be developed that is able to deliver the drug to patient both in open and closed loop as per the requirement. Usages of both open and closed loop as per the survey is shown in table 2. Architectural model of hybrid drug delivery is shown in figure-4 that consists of following components: 


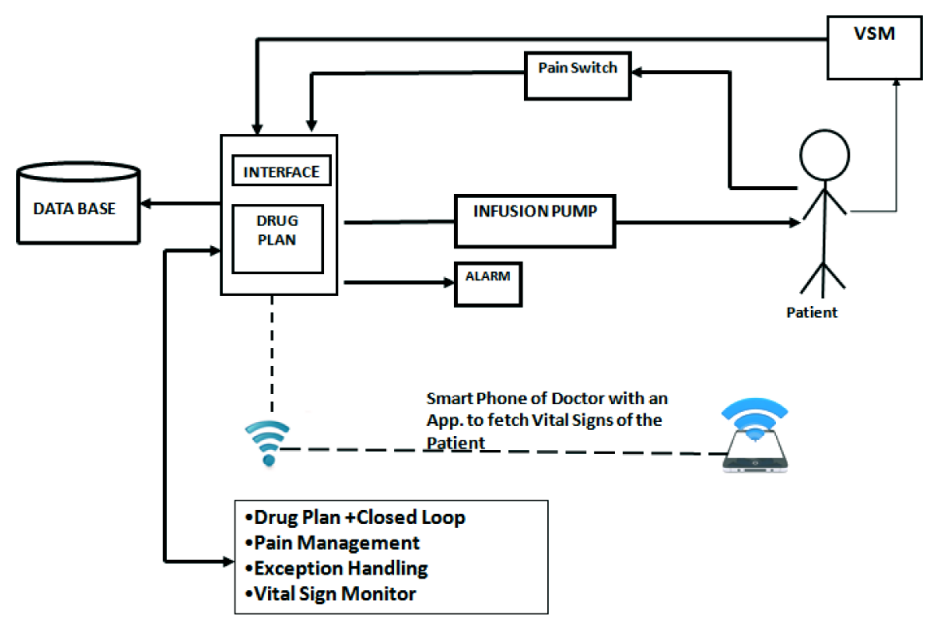

Hybrid Approach for Automatic Drug

Dispensing and Control

Figure 4: That Consists of following Components.

Database: Hybrid drug delivery system consists of a database that helps in storing patient data like name and rate of drug, vital signs of the patient etc. that may help research fellows to analyze case history in future. We design database in MySQL.

Laptop with GUI: Laptop has a User Interface to use the Hybrid Drug Delivery System effectively. This user Interface has been designed in Python or it may also be designed in VB or Java.

Infusion Pump: Infusion pump has a controller that gets the instructions from the laptop.

Vital Sign Monitor: Vital sign monitor is a device connected with patient that sends the vital signs to user interface program as feedback in case of closed loop drug delivery.

Pain Switch: A switch can be connected to bedside of the patient that can be used if he/she has a pain after the surgery.

Alarm: In case the patient presses the switch an alarm will be set a buzz to intimate the nursing staff that the patient has pain. Simultaneously, the system will also send intimation to the doctor's smart phone.

\section{CONCLUSION}

Healthcare technology is constantly changing. However one of the biggest reasons for uptake of a new technology is the Doctor's willingness. If a 
Kumar, $\mathrm{N}$

Panda, SN

Pradhan, $\mathrm{P}$

technology is available but no one is ready to adopt it; then it is of no use. Therefore it is essential to know the willingness of the intended user. Our study has shown that a drug delivery system is required that can be operated both in closed loop and open loop and can transmit vital parameters of the patient to an expert at a remote location. The proposed system has a database that assists the researcher to view the detailed case study (on a minute to minute basis). Doctors can also plan about the drug e.g. name of the drug, rate of the drug, fix up the range of vital signs and action. This system can be used effectively post surgeries in ICU/CCU. It consists of a switch that can be pressed by the patient if he/she is having pain, This information may be sent to the doctor on his/her smart phone that patient has pain. Expert system facility can also be incorporated within hybrid drug delivery system to make it more reliable.

\section{FUTURE SCOPE}

This hybrid drug delivery system may be installed in ambulances because, with this, lifesaving drugs can be effectively provided to a patient, who is being shifted to hospital for further treatment. With the advancement in IOT (Internet of Things) the doctors will be able to control this hybrid drug delivery system from remote location. Thus, virtual presence of a doctor can be provided to save the precious life of a patient. By transmitting the vital parameters of the patient to a doctor at remote location, will be able to reduce the time for initiating treatment and allow the emergency crew to be better prepared. As data is stored in the data base so in future this data can be used as a case study by the researchers.

\section{REFERENCES}

[1] Simanski, O., Schubert, A., Kaehler, R., Janda, M., Bajorat, J., Hofmockel, R. and Lampe, B., 2007, June. Automatic drug delivery in anesthesia: From the beginning until now. In Control \& Automation, 2007. MED’07. Mediterranean Conference on (pp. 1-6). IEEE.

[2] Ziv, Stephen D. Small, Paul Root Wo, A. (2000). Patient safety and simulationbased medical education. Med Teach, 22(5), pp.489-495.

https://doi.org/10.1080/01421590050110777

[3] Arney, D., Pajic, M., Goldman, J.M., Lee, I., Mangharam, R. and Sokolsky, O., 2010, April. Toward patient safety in closed-loop medical device systems. In Proceedings of the 1st ACM/IEEE International Conference on Cyber-Physical Systems (pp. 139-148). ACM https://doi.org/10.1145/1795194.1795214

[4] Huang, J., Ying-Ying Lu, Nayak, A. and Roy, R. (1999). Depth of anesthesia estimation and control [using auditory evoked potentials]. IEEE Transactions on Biomedical Engineering, 46(1), pp.71-81. https://doi.org/10.1109/10.736759 
[5] Kameneva, Tatiana et al. 'A Comparison of Open-Loop And Closed-Loop Stimulation Strategies To Control Excitation Of Retinal Ganglion Cells'. Biomedical Signal Processing and Control 14 (2014): 164-174. Web. https://doi.org/10.1016/j.bspc.2014.07.015

[6] Dumont, Guy A., and J. Mark Ansermino. 'Closed-Loop Control of Anesthesia : A Primer for Anesthesiologists'. Anesthesia \& Analgesia 117.5 (2013): 11301138. Web. https://doi.org/10.1213/ANE.0b013e3182973687

[7] Hayakawa, T., Haddad, W.M., Bailey, J.M. and Hovakimyan, N. (2005) 'Passivity-Based Neural Adaptive Output Feedback Control for Nonlinear Nonnegative Dynamical Systems', IEEE Transaction on Neural Network, vol. 16, March, pp. 387-398. https://doi.org/10.1109/TNN.2004.841782

[8] Lin, H., Beck, C. and Bloom, M. (2004). On the Use of Multivariable PiecewiseLinear Models for Predicting Human Response to Anesthesia. IEEE Transactions on Biomedical Engineering, 51(11), pp.1876-1887. https://doi.org/10.1109/TBME.2004.831541

[9] AGARWAL, J., PURI, G. and MATHEW, P. (2009). Comparison of closed loop vs. manual administration of propofol using the Bispectral index in cardiac surgery. Acta Anaesthesiologica Scandinavica, 53(3), pp.390-397.

https://doi.org/10.1111/j.1399-6576.2008.01884.x

[10] Struys, M., De Smet, T., Versichelen, L., Van de Velde, S., Van den Broecke, R. and Mortier, E. (2001). Comparison of Closed-loop Controlled Administration of Propofol Using Bispectral Index as the Controlled Variable versus "Standard Practice" Controlled Administration. Anesthesiology, 95(1), pp.6-17. https://doi.org/10.1097/00000542-200107000-00007

[11] Soltesz, K., Dumont, G., van Heusden, K., Hagglund, T. and Ansermino, J.M., 2012, December. Simulated mid-ranging control of propofol and remifentanil using EEG-measured hypnotic depth of anesthesia. In Decision and Control (CDC), 2012 IEEE 51st Annual Conference on (pp. 356-361). IEEE.

[12] Meystre, S., 2005. The current state of telemonitoring: a comment on the literature. Telemedicine Journal \& e-Health, 11(1), pp.63-69.

https://doi.org/10.1089/tmj.2005.11.63

[13] Fernandes, B., Afonso, J.A. and Simões, R., 2011, June. Vital signs monitoring and management using mobile devices. In 6th Iberian Conference on Information Systems and Technologies (CISTI 2011) (pp. 1-6). IEEE.

[14] van Halteren, A.T., Bults, R.G.A., Wac, K.E., Konstantas, D., Widya, I.A., Dokovski, N.T., Koprinkov, G.T., Jones, V.M. and Herzog, R., 2004. Mobile patient monitoring: The mobihealth system.

[15] Yuan, B. and Herbert, J., 2011, March. Web-based real-time remote monitoring for pervasive healthcare. In Pervasive Computing and Communications Workshops (PERCOM Workshops), 2011 IEEE International Conference on (pp. 625-629). IEEE. https://doi.org/10.1109/PERCOMW.2011.5766964
Hybrid Approach for Automatic Drug Dispensing and Control 